\title{
Effects of Lexicase and Tournament Selection on Diversity Recovery and Maintenance
}

\author{
Thomas Helmuth \\ Computer Science \\ Washington and Lee $U$. \\ Lexington, Virginia, USA \\ helmutht@wlu.edu
}

\author{
Nicholas Freitag McPhee \\ Div. of Science \& Mathematics \\ $U$. of Minnesota, Morris \\ Morris, Minnesota, USA \\ mcphee@morris.umn.edu
}

\author{
Lee Spector \\ Cognitive Science \\ Hampshire College \\ Amherst, Massacusetts, USA \\ Ispector@hampshire.edu
}

\begin{abstract}
In genetic programming systems, parent selection algorithms select the programs from which offspring will be produced by random variation and recombination. While most parent selection algorithms select programs on the basis of aggregate performance on multiple test cases, the lexicase selection algorithm considers each test case individually, in random order, for each parent selection event. Prior work has shown that lexicase selection can produce both more diverse populations and more solutions when applied to several hard problems. Here we examine the effects of lexicase selection, compared to those of the more traditional tournament selection algorithm, on population error diversity using two program synthesis problems. We conduct experiments in which the same initial population is used to start multiple runs, each using a different random number seed. The initial populations are extracted from genetic programming runs, and fall into three categories: high diversity populations, low diversity populations, and populations that occur after diversity crashes. The reported data shows that lexicase selection can maintain high error diversity and also that it can re-diversify less-diverse populations, while tournament selection consistently produces lower diversity.
\end{abstract}

\section{Keywords}

diversity; lexicase selection; tournament selection; hyperselection; PushGP

\section{INTRODUCTION}

Parent selection is one of the fundamental processes in genetic programming (GP), and several different parent selection algorithms have been developed. Lexicase selection is a relative newcomer on the scene $[13,5]$. While most commonly-used parent selection algorithms, such as tournament selection, select programs on the basis of aggregate performance on multiple test cases, the lexicase selection algorithm considers each test case individually, in random

Permission to make digital or hard copies of all or part of this work for personal or classroom use is granted without fee provided that copies are not made or distributed for profit or commercial advantage and that copies bear this notice and the full citation on the first page. Copyrights for components of this work owned by others than the author(s) must be honored. Abstracting with credit is permitted. To copy otherwise, or republish, to post on servers or to redistribute to lists, requires prior specific permission and/or a fee. Request permissions from permissions@acm.org.

GECCO'16 Companion, July 20 - 24, 2016, Denver, CO, USA

(C) 2016 Copyright held by the owner/author(s). Publication rights licensed to ACM. ISBN 978-1-4503-4323-7/16/07 . \$15.00

DOI: http://dx.doi.org/10.1145/2908961.2931657 order, for each parent selection event. Compared to tournament selection, lexicase selection has been shown to produce more diverse populations and more solutions on several classes of problems, including software synthesis benchmark problems $[5,1,4,2]$. This prior work made connections between high diversity and high solution rates when using lexicase selection. Each of these studies examined the diversity of entire GP runs, each starting with a different initial population and random number seed.

Our work here was originally motivated by observations of dramatic drops in diversity that result from "hyperselection" events when using lexicase selection, in which one or a few individuals in the population receive the majority of the parent selections in a generation [3]. Anecdotally, these runs often seemed to recover diversity within a handful of generations following the diversity crash. Because a capability for recovering adaptive diversity is desirable, we decided to examine the dynamics of diversity after diversity crashes more systematically, producing data from runs using lexicase selection and, for comparison, tournament selection.

While examining the effects of selection algorithms in the wake of diversity crashes is enlightening, such a study has scope limited to infrequent events when using lexicase selection. More broadly, we are interested in the immediate effects on diversity that these algorithms have in populations with other defining characteristics. In particular, we wish to explore how each method influences diversity in populations that occur naturally during evolution, specifically in populations with usually high or low diversity.

In order to study these questions, we conducted sets of runs in which each run was initialized with the same population, with each population extracted from a GP run. This allows us to observe how different selection methods affect diversity when started on the exact same population.

In the next section we describe the two selection algorithms in our study. We then describe our experimental design, including a discussion of the diversity measure that we used (error diversity), the ways in which we extracted initialization populations, the two test problems that we used, and the parameters of our runs. Finally, we present the results of the experiments and discuss their implications.

\section{PARENT SELECTION}

In this section we describe the two parent selection algorithms used in our experiments.

\subsection{Tournament Selection}

We use standard tournament selection as a comparison 
method. In it, we choose random individuals (with replacement) to form a tournament set, and select the individual with the lowest total error to be a parent.

\subsection{Lexicase Selection}

Each time a parent must be selected, lexicase selection first shuffles the list of test cases into a random order. Then, starting with the entire population, it removes any individual that did not achieve the best error value on the first test case. As long as more than one individual remains in the population, the first test case is removed and this process is repeated with the next test case, etc. For each test case, any individual that did not have the best error of those remaining in the pool is removed from consideration. If at any point only a single individual remains, it is selected as the winner of that parent selection event. On the other hand, if every test case is exhausted and multiple individuals remain, one of them is randomly selected. A more detailed description of lexicase selection is given in [5].

Lexicase selection shares some motivation with other recent techniques that consider not how an individual performs across an entire problem in aggregate, but instead how it performs on parts of a problem. This work on "behaviorbased" or "semantic" search operators includes geometric semantic GP [12], behavioral GP [8], clustering test cases into objectives [7, 11], and other behavior-based search drivers [9]. Lexicase selection sets itself apart from these methods by placing importance on individual test cases and combinations of test cases - those test cases that come at the beginning of each random ordering of cases.

\section{EXPERIMENTAL DESIGN}

In this paper we concentrate on diversity measures related to the outputs of the programs. One such diversity measure, behavioral diversity, has been shown to correlate with problem-solving performance [6]. In behavioral diversity, the output of a program on each training case input is recorded as a behavior vector. Behavioral diversity is then the percentage of distinct behavior vectors in the population. Error diversity, a slight variation of behavioral diversity, considers the percentage of distinct error vectors in the population where each error vector is computed by applying the error function to each output in the behavior vector. We believe error diversity does a good job of measuring how well evolution is exploring meaningful differences between programs that might be lost with a diversity measure that only takes into account syntactic (genotypic) diversity of the population, since two wildly different programs may actually compute the same function.

In order to produce the populations on which to experiment, we started GP runs and let them continue until they met certain stopping conditions; we then stored those populations and later conducted multiple trials with different random number seeds starting with those stored populations. We used three different stopping conditions in order to generate naturally occurring populations with interesting properties: high diversity, low diversity, and after a diversity crash.

High diversity: In lexicase selection runs, we stopped if the population error diversity was greater than 0.9. This produces diverse populations, allowing us to observe whether evolution is able to maintain such high diversity in the following generations.
Table 1: PushGP parameters

\begin{tabular}{lr}
\hline Parameter & Value \\
\hline runs per problem/parent selection combination & 100 \\
population size & 1000 \\
maximum genome size & 800 \\
maximum initial genome size & 400 \\
tournament size (for tournament selection) & 7 \\
\hline Genetic Operator & Probability \\
\hline alternation & 0.2 \\
uniform mutation & 0.2 \\
uniform close mutation & 0.1 \\
alternation followed by uniform mutation & 0.5 \\
\hline
\end{tabular}

Low diversity: In runs using tournament selection, we stopped if the population error diversity was less than 0.15 . These populations allow us to see if methods promote diversification starting from such undiverse populations.

After a diversity crash: As described above, we were initially motivated here by observations of runs using lexicase selection that underwent major drops in diversity following hyperselection events. In this condition, we stopped runs using lexicase selection when the error diversity reached a level at least 0.25 less than it had been at some point in the previous 10 generations. This allowed us to detect populations that had recently undergone large drops in diversity. We do not definitively know whether those drops are related to hyperselection events, but we expect that they are.

In all three conditions, we only considered populations occurring after the first 10 generations in order to give evolution a chance to settle down after the extreme shifts that can happen at the beginning of a run.

In each trial, we continued running GP on a stored population for 20 generations while recording the population error diversity. For each parent selection setting (lexicase and tournament selection), we conducted 100 trials with different random number seeds from the same stored population.

We conducted these tests on two problems from a recent program synthesis benchmark suite [4]. The first problem, Replace Space With Newline (RSWN), specifies a target program that takes a string as input and both prints an output string and returns a result. The printed string should be a copy of the input string with all spaces replaced by newline characters. The functionally returned result should be the number of non-whitespace characters in the input string. Previous examinations of error vector diversity on the RSWN problem show that lexicase selection maintains significantly higher diversity than tournament selection [2].

The second problem, Double Letters, requires the production of a program that takes a string as input and prints the string after doubling every alphabetic character and tripling every exclamation point. All other characters should be printed once. As with the RSWN problem, lexicase selection consistently achieves high diversity on this problem. Differently than RSWN, runs using tournament selection show slow but steady increases in diversity, though not approaching that of lexicase selection runs [2].

For our experiments we used PushGP [16, 15], a stackbased GP system. ${ }^{1}$ PushGP supports a variety of control

\footnotetext{
${ }^{1}$ Lexicase selection has also been shown to be effective in tree-based GP $[5,11]$.
} 
structures and multiple data types, making it a good choice for program synthesis tasks such as the problems we explore here. Except for parent selection, we used the exact same PushGP parameters in both the initial runs used to store interesting populations as well as the continuations of the stored populations. We give the most relevant parameters in Table 1. The parameters not listed here exactly follow those described in [1], where the meanings of all parameters are also explained in detail.

These runs use the most recent version of PushGP, in which individuals are stored as linear genomes that we translate into hierarchical Push programs prior to execution [1]. These linear genomes admit a range of uniform genetic operators; we use four, listed in Table 1 with their probabilities. Alternation is a linear crossover operator modeled after the recombinative portion of ULTRA [14]. Uniform mutation may replace each instruction with $1 \%$ probability. Uniform close mutation may add or remove hierarchydelineating parentheses from the program. Finally, the last operator runs alternation on two parents and then uniform mutation on that child to produce a new child.

\section{RESULTS}

Using the techniques presented in the previous section we obtained populations on which to perform continuation experiments. For each combination of the two problems and three stopping conditions we stored populations from two runs, for a total of 12 populations. In the following subsections we group the results based on the stopping conditions, since they produce the most similar populations.

Starting with each stored population we conducted 100 "continuation" GP runs with lexicase selection and 100 with tournament selection. We let each continuation evolve for 20 generations, and plot the population error diversity across the runs. In particular, each figure has a standard box-andwhisker plot for each generation, with the box showing the median and quartiles. The whiskers stretch to the maximum and minimum values, ignoring outliers. In each figure we also plot the error diversity of each individual run at each generation, giving another way of visualizing the spread of diversities across runs.

Note that in a few settings, one or two runs out of 100 found solutions to the problem before the end of 20 generations. In these cases, we terminate the runs, and they do not contribute data past their termination generation.

\subsection{Starting with high diversity}

First, we examine continuations initialized with populations that were evolved using lexicase selection and achieved error diversity greater than 0.9. As such, the initial populations of the continuations have very high diversity, with most individuals producing distinct error vectors.

Figure 1 plots the continued runs started from two populations (A and B) stored from GP on the RSWN problem. Lexicase selection consistently maintains high levels of diversity starting from both populations, with little variance. On the other hand, both plots show runs using tournament selection quickly losing significant diversity within the first 5 to 10 generations of the continuation, dropping from over $90 \%$ distinct error vectors down to around $50 \%$ distinct error vectors. Interestingly, the tournament selection runs on Population A show large differences in diversity in the last 10 generations, with some becoming even less diverse while oth- ers recover much of the lost diversity. On the other hand, the tournament selection runs on Population B maintain much more consistent diversity, with most runs having between $40 \%$ and $60 \%$ diversity in the remaining generations.

Figure 2, which plots the diversities of continuations of two populations $(\mathrm{C}$ and $\mathrm{D})$ on the Double Letters problem, shows similar trends in both lexicase selection and tournament selection. Note that tournament selection maintains higher diversity on this problem than on the RSWN problem, though not as high as lexicase selection. This trend mirrors what has been seen previously on full GP runs [2].

\subsection{Starting with low diversity}

Next, we present continuations of runs that start from populations exhibiting population diversity of at most 0.15 , i.e., most of the individuals in these populations produced the same error vectors. These populations were stored from runs that used tournament selection, since we were not able to achieve population diversity this low in a run using lexicase selection. Additionally, these runs will shed light on whether the parent selection technique used to produce the initial populations has effect on the continued diversity.

Figure 3 plots diversity of runs starting from populations $\mathrm{E}$ and $\mathrm{F}$ on the RSWN problem. In neither case does tournament selection increase diversity across the 20 generations except for a handful of outlier runs. The continuations using lexicase selection increase the median diversity across runs rapidly, with over $50 \%$ unique error vectors by generation 8 using population E and generation 4 using population F. For population E, lexicase selection runs continue to steadily rise in diversity over the 20 generations. On the other hand, many runs starting with population $\mathrm{F}$ undergo steep drops in diversity, such that by generation 9 the lower quartile of diversity falls precipitously from around $60 \%$ to around $35 \%$. The indivdiually plotted run diversities show that many runs continue to see single-generation drops in diversity throughout the 20 generations. We believe this population likely contained one or more individuals that, when varied in the right way, produce a child that dominates the rest of the population, leading to hyperselection events - and therefore drops in diversity - in many runs. Even with these drops in diversity, lexicase selection maintains higher diversity than tournament selection in the majority of continuations.

We present continuations of low-diversity populations ( $G$ and $\mathrm{H}$ ) evolved on the Double Letters problem in Figure 4. Lexicase selection again increases error diversity more quickly than tournament selection, though here tournament selection does show some increases in diversity. This is more pronounced when starting from population $\mathrm{H}$, where median diversity is over 0.25 by generation 20 . This still pales in comparison to lexicase selection's diversity, which grows to more than 0.6 on population $\mathrm{H}$ and 0.75 on population G. Both plots show lexicase selection runs gaining and maintaining diversity across the 20 generations, without any of the diversity drop-offs that we observed in Figure 3.

\subsection{Starting after a diversity crash}

In Figure 5 we plot error diversity from populations I and $\mathrm{J}$ on the RSWN problem, which were stored after diversity crashes of at least $25 \%$. For the lexicase selection runs, neither of these plots shows rapid rediversification; instead, we see consistent gains in diversity for most of each run. The median diversity on population I increases about $20 \%$ over 


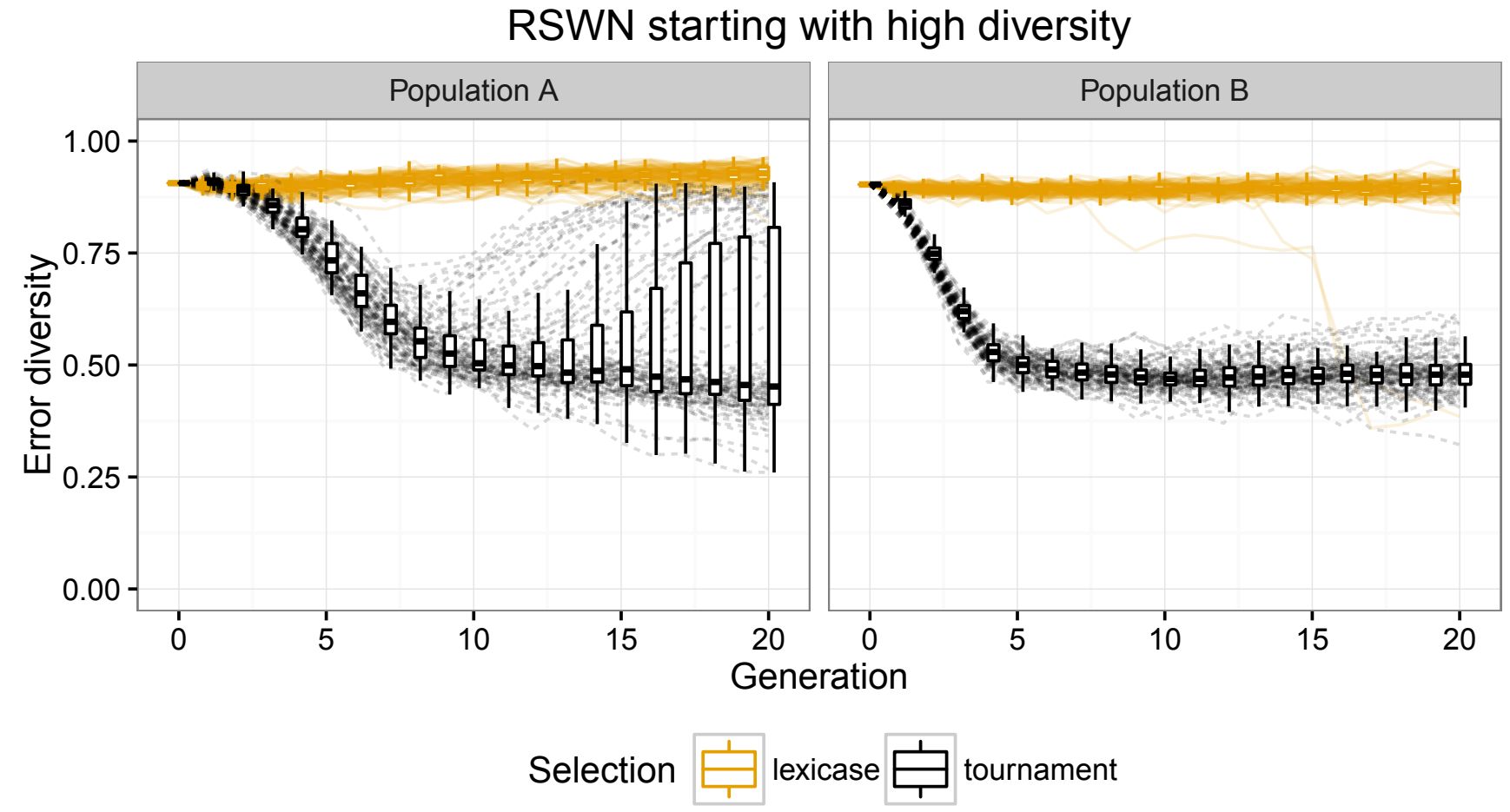

Figure 1: Error diversity over 100 continuations of the RSWN problem with both lexicase and tournament selection, starting from populations with high diversity naturally occuring in a run using lexicase selection.

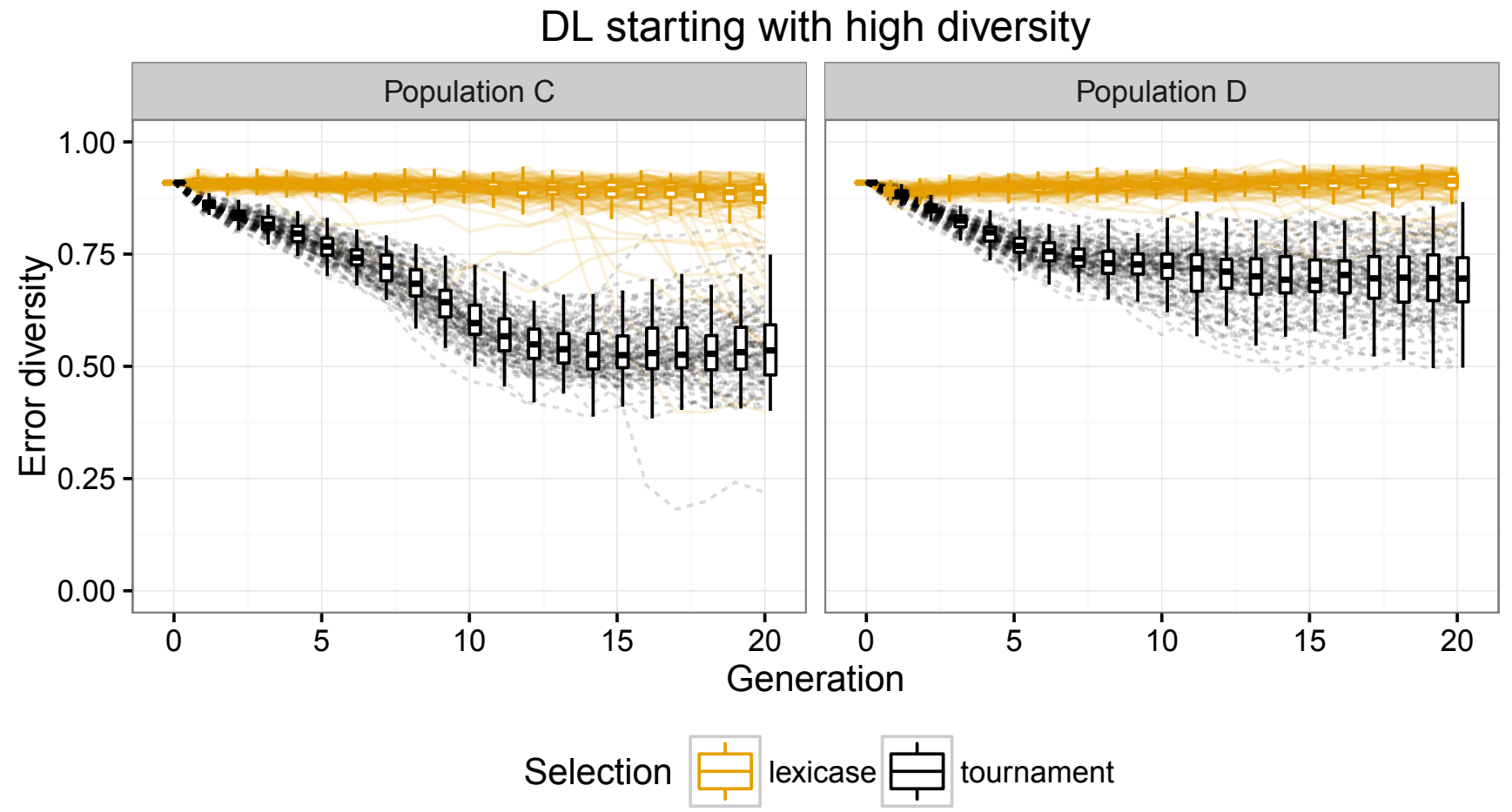

Figure 2: Error diversity over 100 continuations of the double-letters problem with both lexicase and tournament selection, starting from populations with high diversity naturally occuring in a run using lexicase selection. 


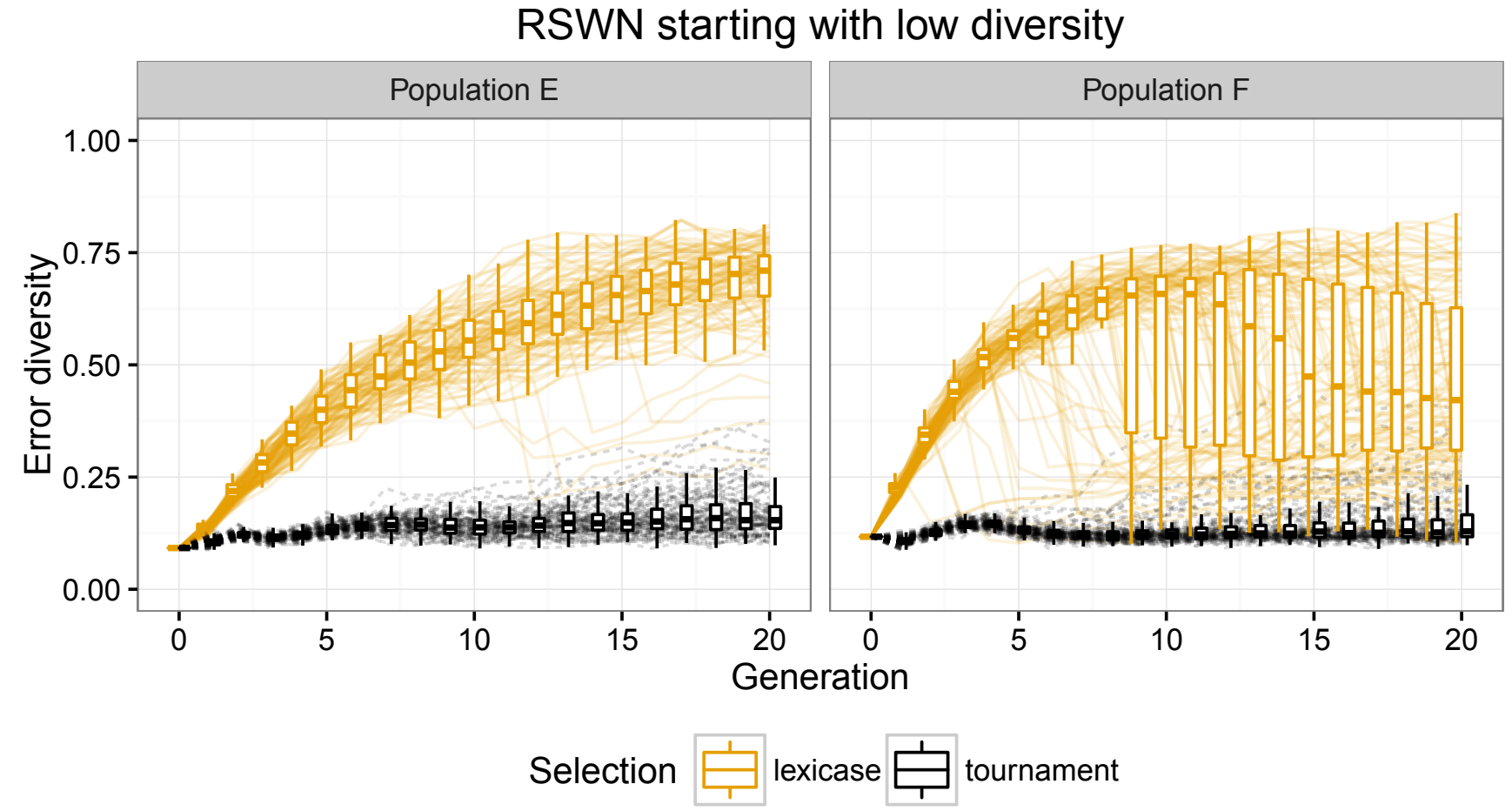

Figure 3: Error diversity over 100 continuations of the RSWN problem with both lexicase and tournament selection, starting from populations with low diversity naturally occuring in a run using tournament selection.

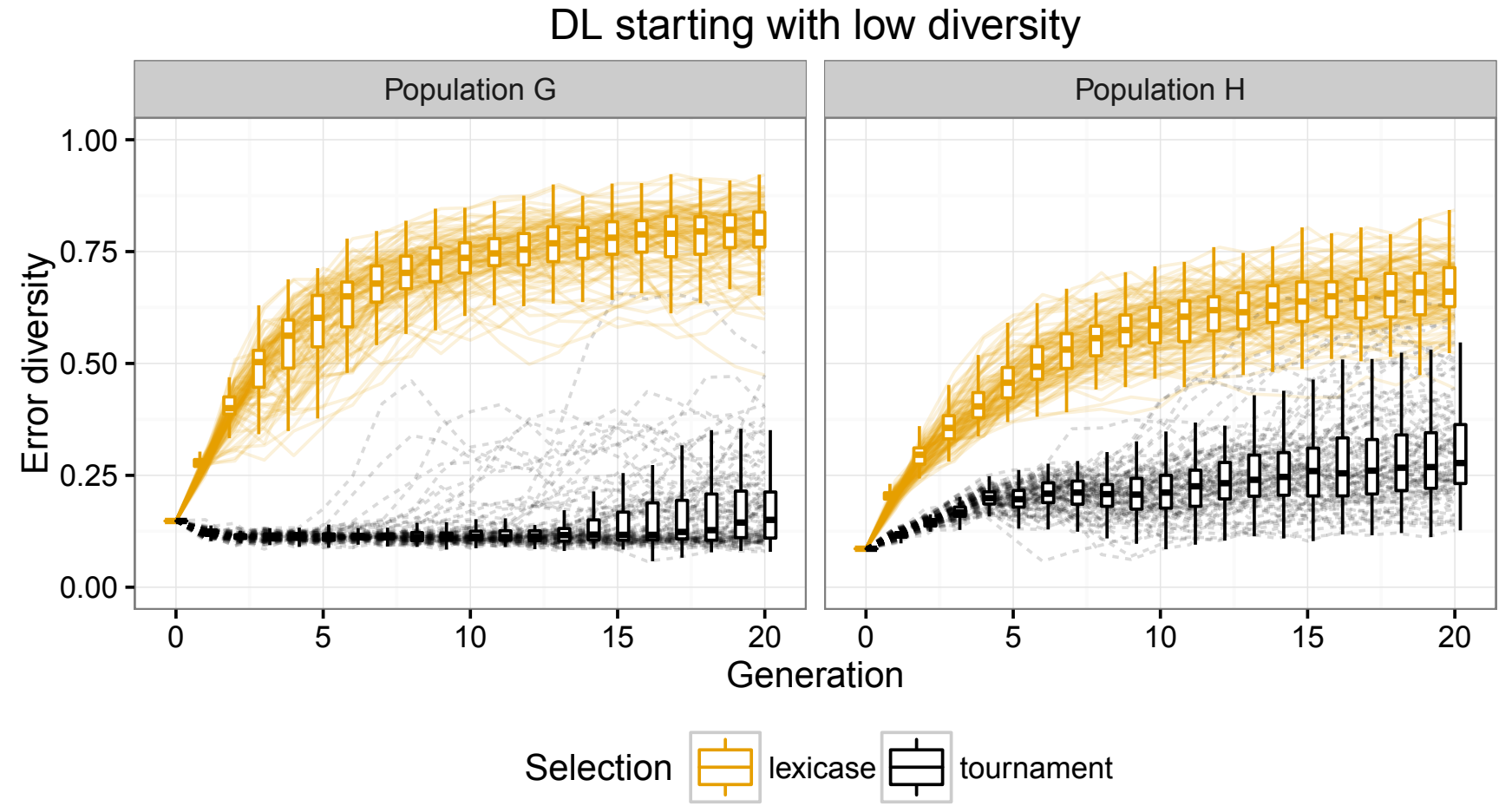

Figure 4: Error diversity over 100 continuations of the double-letters problem with both lexicase and tournament selection, starting from populations with low diversity naturally occuring in a run using tournament selection. 


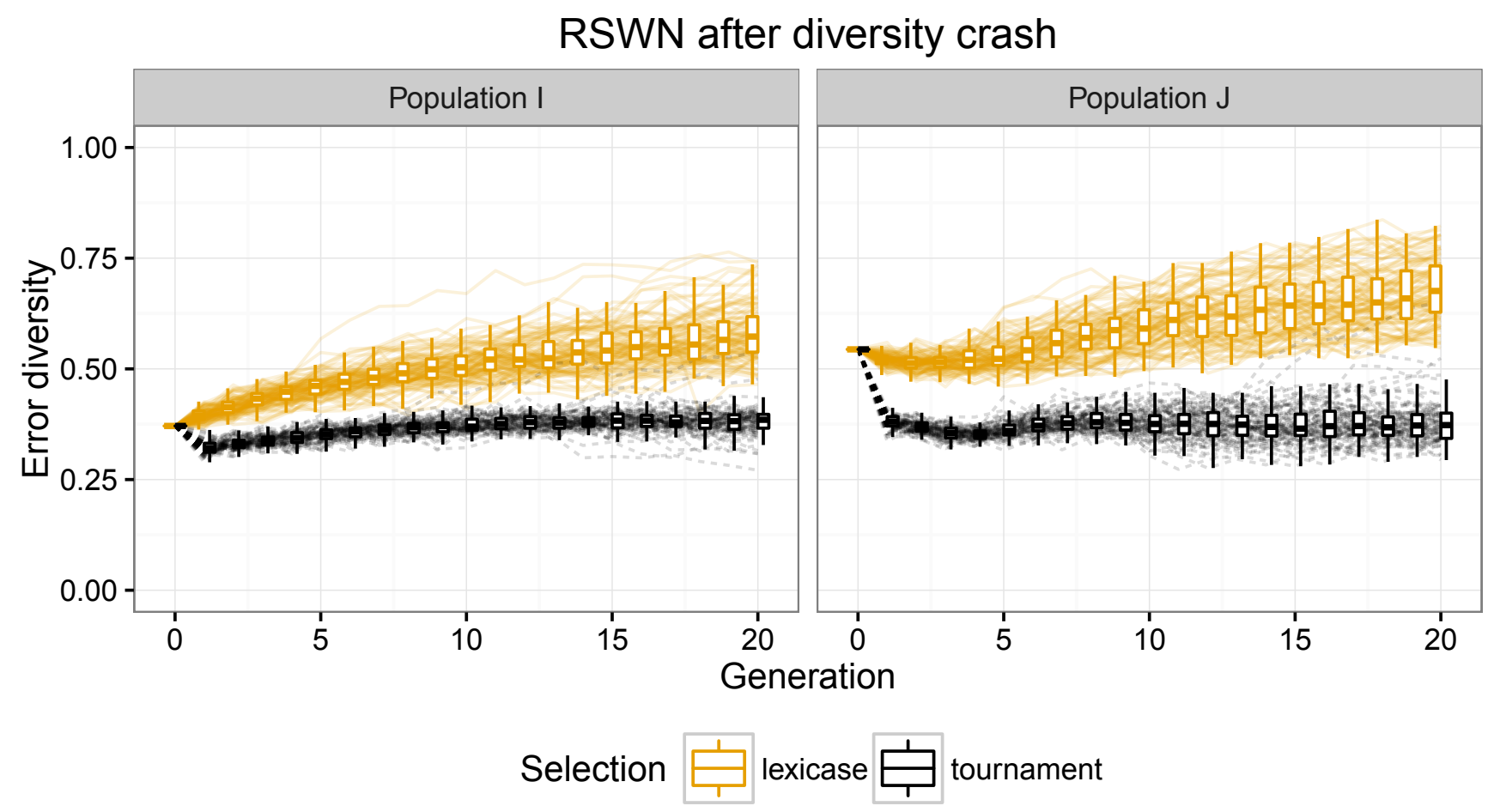

Figure 5: Error diversity over 100 continuations of the RSWN problem with both lexicase and tournament selection, starting from populations that had lost at least $25 \%$ error diversity in a diversity crash in a lexicase selection run.

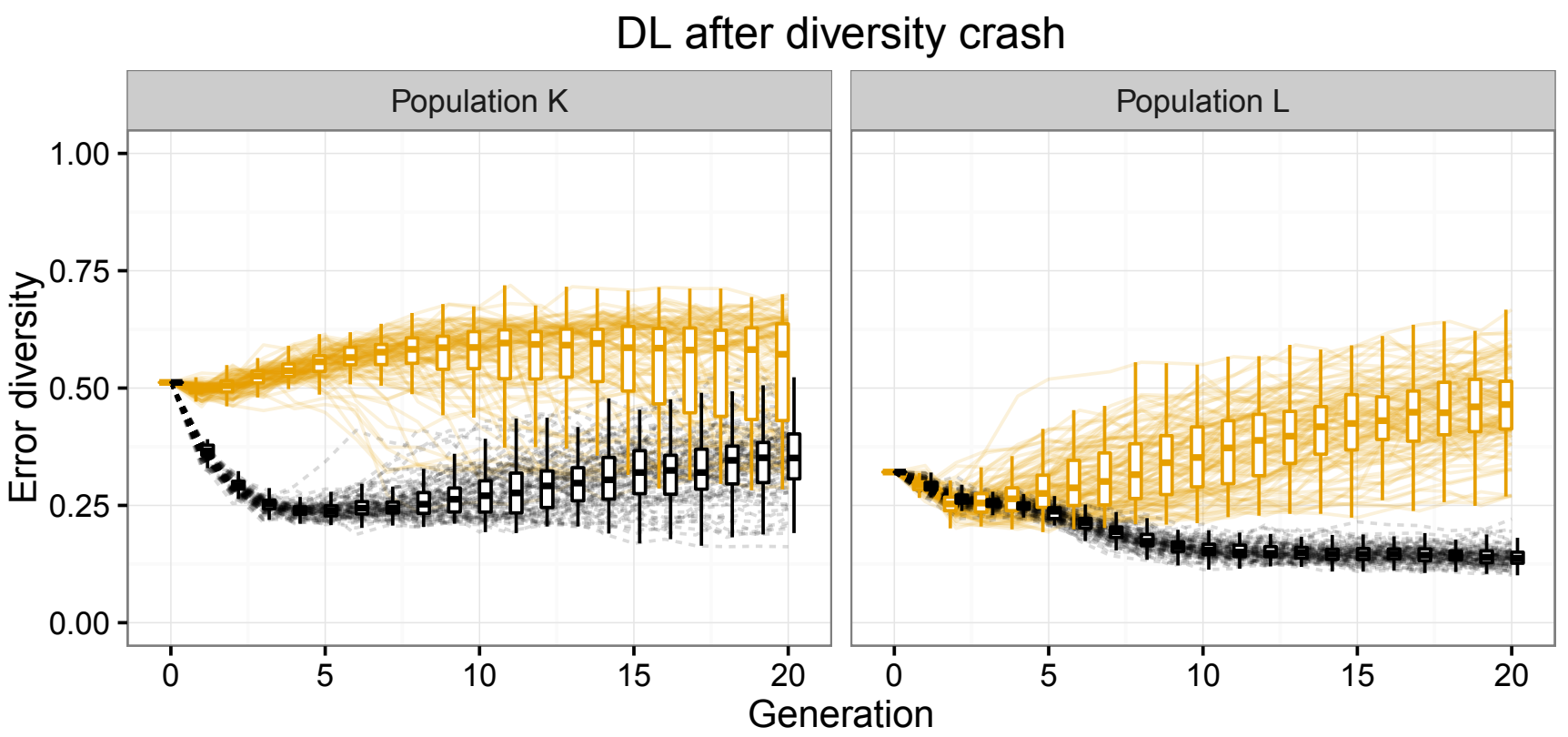

Selection

Figure 6: Error diversity over 100 continuations of the double-letters problem with both lexicase and tournament selection, starting from populations that had lost at least $25 \%$ error diversity in a diversity crash in a lexicase selection run. 
20 generations, gaining back most of the diversity it lost during the diversity crash. Population J gains back about $15 \%$ population in that timespan. Interestingly, population I sees immediate small gains in diversity in the first few generations, whereas population J shows consistent small losses in diversity before rediversifying. This presumably means that population I had reached its minimum in its diversity crash, whereas population $\mathrm{J}$ was recorded near the end of the crash but before its minimum.

Turning our attention to tournament selection, we see that it produced remarkably consistent drops in diversity in the very first generation, especially in Population J. These drops are followed by little movement either direction during the remaining generations.

Figure 6 shows the same settings for the Double Letters problem using populations $\mathrm{K}$ and L. Population $\mathrm{K}$ is interesting in that lexicase selection gains some diversity near the beginning while almost every tournament selection loses at least $25 \%$ diversity over the first 4 generations. After that, an increasing number of lexicase selection runs seem to undergo further diversity crashes, pulling down the lower quartile while the median diversity stays around $60 \%$. On the other hand, tournament selection shows incrases in diversity in the later generations, although its quartiles never overlap with lexicase selection's.

For population L, after a few generations of further diversity decreases, lexicase selection runs tend to increase diversity (with lots of variation) ending with about $20 \%$ higher median diversity than at its lowest. Tournament selection, however, consistently loses diversity over the 20 generations with little variance across runs.

\section{DISCUSSION}

The results from continuations of high-diversity populations clearly show that lexicase selection can maintain a high population diversity while tournament selection cannot reliably do so. One notable feature visible in the initial-highdiversity plots (Figure 1 and Figure 2) is the occasional steep drop in diversity in a small number of runs using lexicase selection, which can be seen especially clearly in populations $\mathrm{B}$ and C. Based on similar runs we have encountered previously, we would guess that these runs underwent hyperselection events in which one or a small number of individuals were selected to make most of the children in a single generation. Hyperselection events can, understandably, lead to diversity crashes since most of the individuals in the population are closely related. Interestingly, previous work has shown that such events are neither a driving force nor a hinderance in runs using lexicase selection [3].

The continuations starting from low-diversity populations (Figures 3 and 4) show how lexicase selection can diversify an un-deriverse population over a small number of generations. This ability to create error diversity helps to explain how lexicase selection can rapidly explore the space of meaningfully different programs.

Of the results we present, those from runs starting with populations that were produced by diversity crashes (Figure 5 and Figure 6) show the smallest gaps in diversity between lexicase selection and tournament selection. Still, they demonstrate lexicase selection's ability to increase diversity, albeit gradually, following a diversity crash. Tournament selection runs lost diversity over the 20 generations in three of the four conditions, showing not only that it was unable to stop the diversity crash, but also that it extended and exacerbated the crash.

In summary, the reported data shows that lexicase selection can maintain high error diversity and also that it can re-diversify less-diverse populations, whether those populations were produced by tournament selection or by diversity crashes with lexicase selection. Tournament selection consistently produced lower diversity, either by decreasing the number of unique error vectors in the population or by failing to increase diversity in un-diverse populations.

The higher diversity seen with lexicase selection does not, for the problems and configurations examined here, come at the expense of problem-solving power - quite the contrary. In data reported elsewhere [4], lexicase selection produced solutions to the Replace Space with Newline problem in 51 out of 100 runs, while tournament selection succeeded in only 8. On the Double Letters problem, lexicase selection produced 6 solutions (in 100 runs), while tournament selection produced none.

Why might lexicase selection be so much better at increasing and maintaining diversity than tournament selection?

One clue might come from tournament selection's drops in diversity in populations that originally evolved using lexicase selection. Suppose that a number of individuals in a population have identical or very similar error vectors, along with low total error. With tournament selection, these individuals might all be selected a number of times in a given generation, leading to a population containing many of their children. Many of those children likely have similar error vectors to their parents, resulting in a less diverse population than the prior one. With the same population, lexicase selection would require those individuals to compete for the same selections, since any individuals with identical error vectors will have equal chance of selection when their best case errors come near the beginning of the randomly shuffled test cases. So, lexicase selection makes an individual "compete" for the selections it is eligible for with those individuals that produce identical error vectors.

Another factor likely at play here is the way in which lexicase selection places emphasis on individuals that perform well on single test cases or combinations of small numbers of test cases. Since an individual must be the absolute best in the population on a test case if it comes first in the shuffled test cases in order for the individual to be selected, lexicase selection can select individuals that specialize on doing well at one or more test cases even if they do poorly at others. This phenomenon, shown to contribute to lexicase selection's success in prior work [1], likely allows lexicase selection to select many different specialists with different error vectors, diversifying the parent pool and therefore the children of the next generation.

The data presented here are also interesting in other respects. For example, they show that patterns of diversification depend not only on the selection method and on the problem being solved, but also on the starting population. In fact, in some conditions it is evident that the starting population was the cause of later swings in diversity that were not manifested in the first few generations; for example, see population A for tournament selection and population $\mathrm{F}$ for lexicase selection. Thus the composition of a population, or specific members of a population, can drastically change the shape of diversity in the following generations, even compared to similarly chosen populations. 
Even so, lexicase selection clearly contributes more to diversity than tournament selection, starting from all twelve of the populations presented here.

\section{CONCLUSIONS}

Previous work showed that lexicase selection often generates and maintains high levels of diversity across separate runs. The experiments presented here demonstrate this phenomenon systematically by conducting multiple runs from the same starting population.

For the two software synthesis benchmark problems studied here, lexicase selection not only maintains high levels of diversity across entire runs, but also reestablishes diversity in many low-diversity populations. The fact that lexicase selection also tends to solve the studied problems more frequently than does tournament selection, which produces less diverse population, strongly suggests that the diversity we are seeing is adaptive diversity that helps the system to explore the search space.

The presented data also shows that there is a sense in which "history is destiny" with respect to diversity in GP. For some pairs of initial populations that were generated from identical conditions (except for random number seeds), distinct patterns of diversity were consistently observed. The choice of selection method has a clear and characteristic effect on diversity, but the nature of the specific starting population can also have consistent, long term effects.

It would be interesting to use the methodology in this paper to compare the diversity produced by other search drivers, such as "discovery of objectives by clustering" [7] and novelty search [10].

\section{Acknowledgments}

Thanks to the members of the Hampshire College Computational Intelligence Lab for discussions that helped shape this work and to Josiah Erikson for systems support. This material is based upon work supported by the National Science Foundation under Grants No. 1129139 and 1331283. Any opinions, findings, and conclusions or recommendations expressed in this publication are those of the authors and do not necessarily reflect the views of the National Science Foundation.

\section{REFERENCES}

[1] T. Helmuth. General Program Synthesis from Examples Using Genetic Programming with Parent Selection Based on Random Lexicographic Orderings of Test Cases. Ph.D. dissertation, 2015.

[2] T. Helmuth, N. F. McPhee, and L. Spector. Lexicase selection for program synthesis: a diversity analysis. In Genetic Programming Theory and Practice XIII, Genetic and Evolutionary Computation. Springer.

[3] T. Helmuth, N. F. McPhee, and L. Spector. The impact of hyperselection on lexicase selection. In GECCO '16: Proceedings of the 2016 Conference on Genetic and Evolutionary Computation, July 2016.

[4] T. Helmuth and L. Spector. General program synthesis benchmark suite. In GECCO '15: Proceedings of the 2015 Conference on Genetic and Evolutionary Computation, July 2015.

[5] T. Helmuth, L. Spector, and J. Matheson. Solving uncompromising problems with lexicase selection.
IEEE Transactions on Evolutionary Computation, 19(5):630-643, Oct. 2015.

[6] D. Jackson. Promoting phenotypic diversity in genetic programming. In PPSN 2010 11th International Conference on Parallel Problem Solving From Nature, volume 6239 of Lecture Notes in Computer Science, pages 472-481, Krakow, Poland, 11-15 Sept. 2010. Springer.

[7] K. Krawiec and P. Liskowski. Automatic derivation of search objectives for test-based genetic programming. In 18th European Conference on Genetic Programming, volume 9025 of LNCS, pages 53-65, Copenhagen, 8-10 Apr. 2015. Springer.

[8] K. Krawiec and U.-M. O'Reilly. Behavioral programming: a broader and more detailed take on semantic GP. In GECCO '14: Proceedings of the 2014 conference on Genetic and evolutionary computation, pages 935-942, Vancouver, BC, Canada, 12-16 July 2014. ACM. Best paper.

[9] K. Krawiec, J. Swan, and U.-M. O'Reilly. Behavioral program synthesis: Insights and prospects. In Genetic Programming Theory and Practice XIII, Genetic and Evolutionary Computation. Springer, 2015.

[10] J. Lehman and K. O. Stanley. Evolving a diversity of virtual creatures through novelty search and local competition. In Proceedings of the 13th Annual Conference on Genetic and Evolutionary Computation, GECCO '11, pages 211-218, New York, NY, USA, 2011. ACM.

[11] P. Liskowski, K. Krawiec, T. Helmuth, and L. Spector. Comparison of semantic-aware selection methods in genetic programming. In GECCO 2015 workshop on Semantic Methods in Genetic Programming. ACM, 2015.

[12] A. Moraglio, K. Krawiec, and C. G. Johnson. Parallel Problem Solving from Nature - PPSN XII: 12th International Conference, Taormina, Italy, September 1-5, 2012, Proceedings, Part I, chapter Geometric Semantic Genetic Programming, pages 21-31. Springer Berlin Heidelberg, Berlin, Heidelberg, 2012.

[13] L. Spector. Assessment of problem modality by differential performance of lexicase selection in genetic programming: A preliminary report. In 1st workshop on Understanding Problems (GECCO-UP), pages 401-408, Philadelphia, Pennsylvania, USA, 7-11 July 2012. ACM.

[14] L. Spector and T. Helmuth. Uniform linear transformation with repair and alternation in genetic programming. In Genetic Programming Theory and Practice XI, Genetic and Evolutionary Computation, chapter 8, pages 137-153. Springer, Ann Arbor, USA, 9-11 May 2013.

[15] L. Spector, J. Klein, and M. Keijzer. The Push3 execution stack and the evolution of control. In GECCO 2005: Proceedings of the 2005 conference on Genetic and evolutionary computation, pages 1689-1696, Washington DC, USA, 2005. ACM Press.

[16] L. Spector and A. Robinson. Genetic programming and autoconstructive evolution with the push programming language. Genetic Programming and Evolvable Machines, 3(1):7-40, Mar. 2002. 